\title{
Perfil bacteriano del shock séptico en una unidad de cuidados intensivos de la altitud del seguro social del Perú
}

\author{
Bacterial profile of septic shock in an intensive care unit of the Peruvian social security \\ altitude
}

Amilcar Tinoco-Solórzano ${ }^{1,2}$, Jorge Chumbes Perez ${ }^{1,3}$, Daniel Molano Franco4, Jorge Luis Vélez-Páez ${ }^{5}$, Antonio Viruez Soto ${ }^{6}$

DOI. 10.21931/RB/2021.06.04.16

Resumen: Conocer el perfil bacteriano del shock séptico permitirá una adecuada elección de antibióticos empíricos. Objetivos: a) Describir el perfil bacteriano del shock séptico en una unidad de cuidados intensivos de la altitud. b) Conocer la localización de los cultivos positivos. c) Identificar la sensibilidad y el mecanismo de resistencia bacteriana. d) Encontrar diferencias de los perfiles bacterianos de la altitud. Estudio retrospectivo transversal. Realizado en una unidad de cuidados intensivos a 3,250 "msnm". Se incluyeron los cultivos positivos y antibiogramas de residentes de la altitud con shock séptico extraídos antes del inicio de los antibióticos durante 7 años. 1,212 muestras. Escherichia coli (18.48\%). Las bacterias gramnegativas presentaron sensibilidad para colistina (94-99\%) el principal mecanismo de resistencia fue betalactamasa de espectro extendido (43-91\%). Staphylococcus aureus (22.19\%). Las bacterias grampositivas presentaron sensibilidad para tigecilina, linezolid (100\%) y vancomicina (36-100\%) el principal mecanismo de resistencia fue ampicilina/sulbactam resistente productor de betalactamasa (50-97\%) y meticilino resistente (87-100\%). En Conclusión. - a) Escherichia coli la gramnegativa más frecuente y Staphylococcus aureus el grampositivo. b) El cultivo más frecuente provenía del tracto respiratorio inferior. c) De las gramnegativas, Pseudomona aeruginosa mostro elevada sensibilidad para colistina, el resto también para tigecilina. El mecanismo de resistencia más frecuente fue betalactamasa de espectro extendido. Las Bacterias grampositivas tienen una elevada sensibilidad para tigecilina, linezolid y vancomicina. Su mecanismo de resistencia más frecuente fue ampicilina/sulbactam resistente. d) No encontramos diferencias de los perfiles bacterianos informados en la altitud. Recomendamos confirmar los resultados de sensibilidad "in vitro" de Tigeciclina.

Palabras clave: Altitud, bacteria, Perú, sepsis, shock, unidad de cuidados intensivos.

\begin{abstract}
Knowing the bacterial profile of septic shock allowed an adequate choice of empirical antibiotics. Objectives: a) To describe the bacterial profile of septic shock in an intensive care unit at altitude. b) Know the location of positive cultures. c) Identify the sensitivity and the mechanism of bacterial resistance. d) Find differences in the bacterial profiles of altitude. Retrospective crosssectional study. Done in an intensive care unit at 3,250 "masl". Positive cultures and antibiograms from high-altitude residents with septic shock taken before antibiotics for 7 years were included. 1,212 samples. Escherichia coli (18.48\%). Gram-negative bacteria showed sensitivity to colistin (94-99\%), the primary resistance mechanism was extended-spectrum beta-lactamase (43-91\%). Staphylococcus aureus (22.19\%). Gram-positive bacteria showed sensitivity to tigecillin, linezolid (100\%), and vancomycin (36-100\%); the primary resistance mechanism was resistant ampicillin/sulbactam producer of beta-lactamase (50-97\%) and resistant methicillin (87-100\%). In Conclusions.- a) Escherichia coli is the most frequent gram-negative and Staphylococcus aureus the gram-positive. b) The most frequent culture came from the lower respiratory tract. c) Of the gram-negative ones, Pseudomonas aeruginosa showed a high sensitivity for colistin, the rest also for tigecillin. The most frequent resistance mechanism was extended-spectrum beta-lactamase. Gram-positive bacteria have a high sensitivity for tigecillin, linezolid, and vancomycin. Its most common resistance mechanism was resistant ampicillin/sulbactam. d) We did not find differences in the reported bacterial profiles at altitude. We recommend confirming the "in vitro" sensitivity results for Tigecycline.
\end{abstract}

Key words: Altitude, bacteria, Perú, sepsis, shock, intensive care units.

\section{Introducción}

El shock séptico tiene una prevalencia del $17.9 \%$, una incidencia del 11.64 al $13.5 \%$ y una mortalidad del $34.5 \%$ del total de ingresos a la "unidad de cuidados intensivos" (UCI)1,2. Del total de cultivos de los pacientes infectados $70 \%$ son positivos $^{3}$. Los cultivos identifican el patógeno y dirigen el tratamiento antibiótico, se recomienda su obtención antes de iniciar antibióticos ${ }^{4}$. Son cultivos positivos; las muestras de pacientes con cuadro clínico compatible con bacteriemia, Si la bacteria no es causa habitual de la contaminación se necesitara al me- nos un cultivo y si la bacteria es causa común de la contaminación se necesitara al menos dos cultivos ${ }^{5}$. El inicio precoz de antibiótico empírico adecuado y eficaz en el shock séptico evita el aumento de su morbi-mortalidad 6 . La elección del antibiótico empírico depende de los antecedentes, del estado clínico del paciente, la localización de la infección y del perfil bacteriológico local que nos informan la prevalencia, susceptibilidad y mecanismo de resistencia 4 .

Definimos: Shock séptico; al estado de hipoperfusión tisu-

Universidad Peruana Los Andes, Facultad de Medicina Humana, Huancayo, Perú.

2 Seguro Social de Salud (ESSALUD), Hospital Nacional Ramiro Priale Priale, Servicio de Cuidados Intensivos e Intermedios, Huancayo, Perú.

${ }^{3}$ Seguro Social de Salud (ESSALUD), Hospital Nacional Ramiro Priale Priale, Servicio de Infectología, Huancayo, Perú.

${ }^{4}$ Hospital San José, Unidad de Cuidados Intensivos, Bogotá, Colombia.

${ }^{5}$ Hospital Pablo Arturo Suárez, Unidad de Terapia Intensiva, Quito, Ecuador.

${ }^{6}$ Hospital del Norte de El Alto, Departamento de Apoyo Crítico, La Paz, Bolivia. 
Lar asociado a sepsis caracterizado por hipotensión o requerimiento de vasopresores para mantener una "presión arterial media" (PAM) $\geq 65 \mathrm{mmHg}$ y un lactato sérico mayor de 2 $\mathrm{mmol} / \mathrm{L}^{4,7}$. Perfil bacteriano; al reconocimiento de la bacteria responsable de la infección diagnosticado por cultivo. Sensibilidad antimicrobiana; respuesta de la bacteria a los antibióticos determinada a través del antibiograma. Resistencia bacteriana; mecanismo mediante el cual la bacteria puede disminuir la acción de los antibióticos y se estudia a través del antibiogra$\mathrm{ma}^{8}$. Residente de la altitud; todo poblador que se encuentra viviendo en forma constante durante un año como minino por encima de los 1,500 "metros sobre el nivel del mar" (msnm) ${ }^{9}$.

A nivel mundial, Vincent et al (2009) evaluó 14,414 pacientes en 1.265 "UCls" de 75 países, aislando "bacterias gramnegativas" (BGN) (62\%), "bacterias grampositivas" (BGP) (47\%) y hongos (19\%). Los gérmenes más comunes fueron Staphylococcus aureus, Pseudomonas, Escherichia coli, Klebsiella, Staphylococcus epidermidis y Acinetobacter species ${ }^{3}$.

En Latinoamérica, en "UCls" ubicados por debajo de 1,500 "msnm" se encontraron cuatro estudios, ellas reportaron una mayor frecuencia de "BGN". Molina et al. (2011- Colombia) estudio 826 pacientes y Gómez-Gonzales et al. (2018 - Pereira Colombia) estudio 62 pacientes encontrando como bacterias más habituales: Escherichia coli, Staphylococcus aureus, Klebsiella pneumoniae, Pseudomona aeuriginosa y Enterobacter cloacae. En Perú Paz Rojas et al. (2008 - Lima) aisló 1,322 bacterias y Fernández-Mogollón et al. (2016 - Chiclayo) estudio 125 muestras reportando que las bacterias más frecuentes fueron Staphylococcus aureus, Pseudomona aeruginosa, Acinetobacter baumanii, Klebsiella pneumoniae, Staphylococcus epidermidis, Enterococcus faecalis, Enterococcus faecium y Stenotrophomonas maltophila. Klebsiella pneumoniae fue sensible a imipenem, ertapenem, meropenem amikacina trimetoprim/sulfametoxazol y cefepime y resistente a ampicilina/sulbactam, piperacilina/tazobactam y cefazolina. Pseudomona aeruginosa fue sensible a meropenen, y tobramicina y resistencia para ceftazidima, cefepime, amikacina y piperacilina/tazobactam. Escherichia coli fue sensible a ertapenem, meropenem, amikacina y tobramicina y resistente a ampicilina, cefazolina, piperacilina/tazobactam y trimetoprim/sulfametoxazol. Acinetobacter Baumanii fue sensible a imipenem, meropenem y ampicilina/sulbactam ${ }^{10-13}$.

En Latinoamérica, en "UCls" ubicados por encima de los 1,500 "msnm" se encontraron tres estudios encontrando que las bacterias más frecuentes eran las "BGN". Briceño et al. (2006 - Mérida; Venezuela) 1,630 "msnm", estudio 241 muestras, Quintanilla Chanez et al. (2011- Cochabamba, Bolivia) 2,558 "msnm", estudio 40 pacientes y Álvarez et al. (2006 Bogotá, Colombia) 2,640 "msnm", estudio 18,407 microorganismos. Reportando que las bacterias más frecuentes fueron Klebsiella pneumoniae, Pseudomona aeruginosa, Acinetobacter baumannii, Staphylococcus aureus, Escherichia coli y Staphylococcus epidermidis. Klebsiella pneumoniae fue sensible a imipenem y resistente a ciprofloxacina, gentamicina y ceftazidima. Pseudomona aeuroginosa fue sensible a imipenem y resistente a ampicilina/sulbactam, ceftriaxona, cefotaxime, ceftriaxone, gentamicina y amikacina.; Acinetobacter baumanii fue sensible a imipenem y ampicilina/sulbactam y resistente a ciprofloxacina y amikacina. Staphylococcus Aureus fue sensible a vancomicina y resistente a gentamicina, cefotaxima, eritromicina, oxacilina y clindamicina. Echerichia coli; fue sensible a trimetoprim/sulfametoxazol, ampicilina/sulbactam, cefazolina, ciprofloxacina, levofloxacina, y resistente a ampicilina ${ }^{14,15}$. En Latinoamérica no ubicamos estudios ejecutados en "UCls" por encima de los 3000 "msnm".
Nuestros objetivos son: a) Describir el perfil bacteriano de los pacientes con shock séptico en una unidad de cuidados intensivos de un hospital de la altitud del "seguro social del Perú" (EsSalud). b) Conocer la localización de la muestra más frecuente de los cultivos positivos. c) Identificar la sensibilidad y el mecanismo de resistencia bacteriana a los antibióticos más frecuente y d) Encontrar diferencias de los perfiles bacterianos de la altitud y del nivel del mar.

\section{Materiales y métodos}

Estudio observacional, descriptivo, retrospectivo y transversal. Realizado en la "UCl" del "Hospital Nacional Ramiro Priale Priale" de "EsSalud" con seis camas, ubicado en Huancayo a 3,250 "msnm" con una presión barométrica de 535 $\mathrm{mmHg}$. Se analizaron los resultados de los cultivos y antibiogramas de un periodo de 7 años (2012-2018).

Se incluyeron los informes de los cultivos positivos y antibiogramas de los pacientes mayores de 18 años residentes de la altitud con shock séptico extraídos antes del inicio de los antibióticos, al ingreso a la "UCl". Se excluyeron los pacientes con otros tipos de shock asociados, informes de muestras repetidas y los informes incompletos. Para determinar cultivo positivo y susceptibilidad de los antibiogramas se siguieron las recomendaciones de la "Sociedad Española de Enfermedades Infecciosas y Microbiología Clínica" (SEIMC) ${ }^{5}$. Las variables estudiadas fueron: shock séptico, perfil bacteriano, sensibilidad antimicrobiana y resistencia bacteriana. El procedimiento de recolección se inició con la identificación y confirmación de los casos que ingresaron a la "UCl" con shock séptico, luego de aplicar los criterios de inclusión y exclusión se revisaron las historias clínicas de los casos seleccionados para encontrar y analizar los informes de los cultivos y antibiogramas. Para el estudio de los cultivos y del antibiograma se utilizó un equipo VITEK 2-COMPAC de BIOMERIEUX con una autoclave, una incubadora y una refrigeradora.

El tamaño de la muestra calculada para siete años fue de 1,253 y fue determinado con una población de Huancayo de 545,615, una población con "EsSalud" de 147,470 que el promedio de ingresos a la "UCl" por año fue 354, que el shock séptico tiene una prevalencia puntual del 17,9\% y una incidencia calculada del $13,5 \%$, con un nivel de confianza del $95 \%$ y precisión del $5 \%{ }^{1,2}$. Se realizo un muestreo no probabilístico de casos consecutivos.

El análisis estadístico descriptivo univariado, evaluamos la normalidad de las variables cuantitativas numéricas usando métodos gráficos y estadísticos (shapiro wilks y Kolmogorov Smirnov) Las variables con normalidad se analizaron con media y desviación estándar, las variables sin normalidad se analizaron con mediana y rangos. Las variables cualitativas categóricas fueron analizadas utilizando frecuencias y porcentajes. El programa estadístico utilizado fue stata 14 oficial.

El estudio no genero riesgos, no se tuvo contacto directo con los pacientes, se basó en el análisis retrospectivo de resultados de laboratorio. Se codifico las historias clínicas y los resultados de laboratorio asegurando la confidencialidad y anonimato de los sujetos que ingresaron al estudio. Para la ejecución de la investigación se obtuvieron los permisos institucionales y aprobación de los comités de ética del Hospital Nacional Ramiro Priale Priale y de la Facultad de Medicina Humana de la Universidad Peruana Los Andes. Los autores autofinanciaron el estudio. 


\section{Resultados}

Se identificaron 1,526 cultivos positivos con antibiograma de pacientes con shock séptico, luego de aplicar los criterios de exclusión quedaron 1,212 muestras de 826 pacientes. El principal motivo de exclusión fue muestra repetida. Se recolecto los cultivos durante el segundo semestre del 2019. Del 2012 al 2018 se observó un incremento progresivo del 39\% de cultivos positivos, hay una proporción mayor del sexo masculino, una media de 70 años. Las "BGN" fueron las más frecuentes (58.25\%). La bacteria gramnegativa más frecuente fue Escherichia coli (18.48\%). La "BGP" más frecuente fue Staphylococcus aureus (22.19\%). Las muestras más frecuentes provenían del tracto respiratorio inferior (aspirado traqueal y bronquial) (46.2\%). Las muestras de cavidad abdominal (6.19\%) provenían del estudio de líquido peritoneal, absceso intraabdominal, punción de páncreas y punción de hígado. Las muestras de piel (4.62\%) provenían del estudio de herida operatoria obtenida durante la cura quirúrgica. (Tabla 1).

En el tracto respiratorio inferior las bacterias más frecuentes fueron Staphylococcus aureus (28.4\%), Acinetobacter Baumannii (18 \%) y Escherichia Coli (14.8\%). En Sangre (Hemocultivo) los más frecuentes fueron Staphylococcus aureus (22.9\%), Staphylococcus epidermidis (21.8\%) y Escherichia Coli (15,3\%). (Tabla 2).

Las "BGP" (a excepción de los enterococos) presentaron sensibilidad mayor al 90\% para tigecilina, linezolid y vancomicina. Las "BGN" presentaron sensibilidad mayor al 90\% para colistina.

Del total de muestras de Staphylococcus aureus (269): se estudiaron 266 para tigecilina, encontrando 100\% de sensibilidad, se estudiaron 257 para linezolid encontrando $100 \%$ de sensibilidad y se estudiaron 269 para vancomicina encontrando $99 \%$ de sensibilidad. Del total de muestras de Escherichia coli (224) se estudiaron 95 para tigecilina encontrando $99 \%$ de sensibilidad, se estudiaron 95 para colistina encontrando $99 \%$ de sensibilidad y se estudiaron 222 para imipenem encontrando $95 \%$ de sensibilidad (Tabla 3 y 4).

Los mecanismos de resistencia más frecuentes de las "BGP" están relacionado a la producción de enzimas hidrolíticas inhibitorias en el caso de la resistencia a la ampicilina/sulbactam resistente y a la producción de PBP (Penicilin Binding Protein) en el caso de la resistencia a la meticilina. (Tabla 5).

Los principales mecanismos de resistencia de las "BGN" son los relacionados a las betalactamasas de espectro extendido (Tabla 6).

\section{Discusión}

Lo reportado mundialmente no cambian a una altitud mayor a 3,000 "msnm", el mayor porcentaje son "BGN"16 Staphylococcus aureus, Escherichia coli, Acinetobacter baumannii, Klebsiella pneumoniae, Pseudomona aeruginosa y Staphylococcus epidermidis son responsables del $80 \%$ de las infecciones. La infección del tracto respiratoria fue el más frecuente.

\section{Staphylococcus aureus}

"BGP" más frecuente. La prevalencia en 1,400 "UCls" europeas fue del $30 \%$ y de estos $70 \%$ fueron causados por "Staphylococcus aureus meticilino resistente" (MRSA). Nuestra prevalencia fue $22.19 \%$ de Staphylococcus aureus y de estos $87 \%$ son "MRSA". La Prevalencia de "MRSA" en "UCl" oscila entre $8 \%$ y $14 \%$, nuestra prevalencia fue de $19 \%$ del total de 1,212 muestras. La incidencia de "MRSA" en la "UCl" han aumentado desde el 1961. En Estados Unidos entre 1989 y 2002, la proporción de "MRSA" en "UCl" aumentó del 29\% al 60\% convirtiéndose en un patógeno común en las " $\mathrm{UCl}$ " a nivel mundial. Weiner y Tanriover encontraron una mayor frecuencia del Staphylococcus aureus en infecciónes en: catéter venoso central (51\%), tracto urinario (52\%), neumonías asociadas al ventilador (42\%) y del sitio quirúrgico (43\%). Nosotros encontramos frecuencias elevadas en el tracto respiratorio inferior pero mucho menor en sangre y catéter venoso central. Vancomicina sigue siendo un antibiótico eficaz, en los últimos años, fenotipos de resistencia adicionales han llevado a la introducción acelerada de agentes como linezolid y tigeciclina que muestran una mayor tasa de curación microbiológica e inhibición. Nuestros resultados mostraron una elevada sensibilidad para tigecilina, Linezolid, vancomicina y sulfametoxazol/trimetropim lo cual los mantiene como buenas opciones terapéuticas. Ciprofloxacino no es una buena opción por su muy baja sensibilidad contrario a lo reportado en otros estudios. Encontramos que el mecanismo de resistencia más frecuente fue ampicilina/sulbactam resistente, también reportamos dos casos de "Staphylococcus aureus intermedio a vancomicina" (VISA) 17,18

\section{"Staphylococcus Coagulasa Negativo" (ECN)}

Encontramos a Staphylococcus epidermidis, Staphylococcus hominis y Staphylococcus haemolyticus, similar a reportes anteriores. Son frecuentes en pacientes inmunosuprimidos con afectación de la continuidad de la piel por accesos vasculares como catéter venosos centrales, su presencia en los hemocultivos está muy relacionado con contaminación en el momento de la punción. Nuestros resultados muestran elevada sensibilidad a tigecilina, linezolid y vancomicina similar a lo reportado. Encontramos mecanismos de resistencia elevados para ampicilina/sulbactam y meticilino resistente, superior a otros estudios similares que van del 50 al $80 \%{ }^{19,20}$.

\section{Enterococos}

Encontramos Enterococo faecium y Enterococo faecalis similar a reportes latinoaméricanos. Los encontramos con mayor frecuencia en orina, informes anteriores lo informan con mayor frecuencia en cavidad abdominal. Nuestros resultados muestran elevada sensibilidad a tigecilina y linezolid similar a estudios anteriores, convirtiéndolos en buenas opciones terapéuticas sobretodo para "Enterococcus resistente a la vancomicina" (VRE). Los mecanismos de resistencia encontrados son elevada resistencia a meticilina, ampicilina/sulbactan (mayor a los antecedentes) y vancomicina. A nivel mundial se reportan enterococos vancomicina resistentes de hasta el $72 \%$ e incremento en la resistencia a la ampicilina ${ }^{12,21,22}$.

Las "BGN" se ubicaron con mayor frecuencia en el tracto respiratorio inferior y la orina. Las "BGN" no fermentadores encontrados fueron Acinetobacter Baumanni y Pseudomona auriginosa. Excepto la Pseudomona auriginosa (que tuvo buena sensibilidad para colistina), los otros también tuvieron buena sensibilidad para tigecilina y meropenem. Su mecanismo de resistencia más frecuente fue "Betalactamasa de espectro extendido" (BLEE) y las carbapenemasa.

\section{Escherichia coli}

"BGN" más frecuente. Su presencia en el tracto respiratorio inferior concuerda con el aumento de microorganismos no habituales en la neumonía adquirida en la comunidad. En orina es frecuente. Reportamos elevada sensibilidad para tigecilina, 


\begin{tabular}{|c|c|c|}
\hline & $\begin{array}{l}\text { Frecuencia } \\
\qquad(\#)\end{array}$ & $\begin{array}{c}\text { Porcentaje } \\
\quad(\%)\end{array}$ \\
\hline EDAD & 70 & $18-96$ \\
\hline \multicolumn{3}{|l|}{ AÑO } \\
\hline 2012 & 87 & 7.18 \\
\hline 2013 & 155 & 12.79 \\
\hline 2014 & 53 & 4.37 \\
\hline 2015 & 142 & 11.72 \\
\hline 2016 & 212 & 17.49 \\
\hline 2017 & 222 & 18.32 \\
\hline 2018 & 341 & 28.14 \\
\hline \multicolumn{3}{|l|}{ SEXO } \\
\hline Femenino & 326 & 39.47 \\
\hline Masculino & 500 & 60.53 \\
\hline \multicolumn{3}{|l|}{ TIPO DE MUESTRA } \\
\hline Tracto respiratorio inferior & 560 & 46.2 \\
\hline Sangre & 170 & 14.03 \\
\hline Orina & 165 & 13.61 \\
\hline Catéter Venoso central & 120 & 9.9 \\
\hline Cavidad abdominal & 75 & 6.19 \\
\hline Piel & 56 & 4.62 \\
\hline Líquido cefalorraquídeo & 35 & 2.89 \\
\hline Liquido pleural & 15 & 1.24 \\
\hline Heces & 13 & 1.07 \\
\hline Otros & 3 & 0.25 \\
\hline \multicolumn{3}{|l|}{ GRAM } \\
\hline Negativo & 706 & 58.25 \\
\hline Positivo & 506 & 41.75 \\
\hline \multicolumn{3}{|l|}{ BACTERIAS } \\
\hline Staphylococcus aureus & 269 & 22.19 \\
\hline Escherichia coli & 224 & 18.48 \\
\hline Acinetobacter baumannii & 150 & 12.38 \\
\hline Klebsiella pneumoniae & 134 & 11.06 \\
\hline Pseudomonas aeruginosa & 109 & 8.99 \\
\hline Staphylococcus epidermidis & 85 & 7.01 \\
\hline Enterococcus faecium & 36 & 2.97 \\
\hline Staphylococcus hominis & 32 & 2.64 \\
\hline Enterococcus faecalis & 31 & 2.56 \\
\hline Staphylococcus haemolyticus & 29 & 2.39 \\
\hline Otros & 113 & 9.33 \\
\hline $\mathrm{n}=1212$ & & \\
\hline
\end{tabular}

Table 1. Características generales del perfil bacteriano. 


\begin{tabular}{|c|c|c|c|c|c|c|c|c|c|c|c|}
\hline \multirow[t]{3}{*}{ Bacteria } & TRI & S & 0 & CVC & CA & $\mathbf{P}$ & LCR & LP & H & Ot & Total \\
\hline & \# & \# & \# & \# & \# & \# & \# & \# & \# & \# & \# \\
\hline & $\%$ & $\%$ & $\%$ & $\%$ & $\%$ & $\%$ & $\%$ & $\%$ & $\%$ & $\%$ & $\%$ \\
\hline Staphylococcus & 159 & 39 & 5 & 32 & 8 & 16 & 5 & 3 & 1 & 1 & 269 \\
\hline Aureus & 28.4 & 22.9 & 3.0 & 26.7 & 10.7 & 28.6 & 14.3 & 20.0 & 7.7 & 33.3 & 22.2 \\
\hline Escherichia & 83 & 26 & 61 & 8 & 25 & 13 & 2 & 1 & 5 & 0 & 224 \\
\hline Coli & 14.8 & 15.3 & 37.0 & 6.7 & 33.3 & 23.2 & 5.7 & 6.7 & 38.5 & 0.0 & 18.5 \\
\hline Acinetobacter & 101 & 10 & 14 & 8 & 2 & 10 & 3 & 2 & 0 & 0 & 150 \\
\hline Baumannii & 18.0 & 5.9 & 8.5 & 6.7 & 2.7 & 17.9 & 8.6 & 13.3 & 0.0 & 0.0 & 12.4 \\
\hline Klebsiella & 76 & 8 & 16 & 8 & 11 & 7 & 2 & 1 & 3 & 2 & 134 \\
\hline Pneumoniae & 13.6 & 4.7 & 9.7 & 6.7 & 14.7 & 12.5 & 5.7 & 6.7 & 23.1 & 66.7 & 11.1 \\
\hline Pseudomonas & 64 & 3 & 20 & 7 & 8 & 4 & 3 & 0 & 0 & 0 & 109 \\
\hline Aeruginosa & 11.4 & 1.8 & 12.1 & 5.8 & 10.7 & 7.1 & 8.6 & 0.0 & 0.0 & 0.0 & 9.0 \\
\hline Staphylococcus & 3 & 37 & 1 & 26 & 6 & 2 & 6 & 4 & 0 & 0 & 85 \\
\hline Epidermidis & 0.5 & 21.8 & 0.6 & 21.7 & 8.0 & 3.6 & 17.1 & 26.7 & 0.0 & 0.0 & 7.0 \\
\hline Enterococcus & 8 & 2 & 14 & 5 & 4 & 0 & 3 & 0 & 0 & 0 & 36 \\
\hline Faecium & 1.4 & 1.2 & 8.5 & 4.2 & 5.3 & 0.0 & 8.6 & 0.0 & 0.0 & 0.0 & 3.0 \\
\hline Staphylococcus & 5 & 20 & 0 & 3 & 2 & 0 & 1 & 1 & 0 & 0 & 32 \\
\hline Homini & 0.9 & 11.8 & 0.0 & 2.5 & 2.7 & 0.0 & 2.9 & 6.7 & 0.0 & 0.0 & 2.6 \\
\hline Enterococcus & 5 & 1 & 19 & 3 & 2 & 0 & 1 & 0 & 0 & 0 & 31 \\
\hline Faecalis & 0.9 & 0.6 & 11.5 & 2.5 & 2.7 & 0.0 & 2.9 & 0.0 & 0.0 & 0.0 & 2.6 \\
\hline Staphylococcus & 4 & 10 & 3 & 8 & 1 & 1 & 2 & 0 & 0 & 0 & 29 \\
\hline Haemolyticus & 0.7 & 5.9 & 1.8 & 6.7 & 1.3 & 1.8 & 5.7 & 0.0 & 0.0 & 0.0 & 2.4 \\
\hline Otros & 52 & 14 & 12 & 12 & 6 & 3 & 7 & 3 & 4 & 0 & 113 \\
\hline & 9.3 & 8.3 & 7.3 & 10.0 & 8.0 & 5.4 & 20.0 & 20.0 & 30.8 & 0.0 & 9.3 \\
\hline Total & 560 & 170 & 165 & 120 & 75 & 56 & 35 & 15 & 13 & 3 & 1212 \\
\hline & 100 & 100 & 100 & 100 & 100 & 100 & 100 & 100 & 100 & 100 & 100 \\
\hline
\end{tabular}

TRI: Tracto respiratorio inferior, S: Sangre, O: Orina, CVC: catéter venoso central, CA. Cavidad abdominal, P: Piel, LCR: Líquido cefalorraquídeo, LP: Liquido pleural, H: Heces, Ot: Otros.

Tabla 2. Frecuencia de las bacterias según tipo de muestra.

colistina, imipenem, meropenem y ertapenem. Los carbapenémicos se reservan para el shock séptico por Escherichia coli productor de "BLEE". El principal mecanismo de resistencia fue "BLEE" muy superior a lo reportado ${ }^{10,23,24}$.

\section{Klebsiella pneumoniae}

Su presencia en el tracto respiratorio inferior y en el tracto urinario está relacionado a cepas hipervirulenta. Reportamos una elevada sensibilidad para tigecilina, colistina, imipenem, meropenem y ertapenem. Tigecilina antimicrobiano bacteriostático de amplio espectro se usa en infecciones complicadas de piel, tejidos blandos, neumonía adquirida en la comunidad y de infecciones intrabdominales debidas a Klebsiella pneumoniae carbapenemasa. Su uso en infecciones del tracto urinario es limitado. Colistina es una opción frente a una Klebsiella pneumoniae carbapenemasa de resistencia extendida. ceftazidima o cefepime no son opciones empíricas por su elevada resistencia. El mecanismo de resistencia más detectado fue
"BLEE" muy superior a los reportados. En el nivel de resistencia, encontramos Klebsiella pneumoniae con "resistencia extendida" (XDR) que son inferiores a otros estudios ${ }^{10,25,26}$.

\section{Acinetobacter Baumanni}

Su presencia en tracto respiratorio inferior está relacionada con edad avanzada, enfermedad pulmonar crónica, inmunosupresión y uso de antimicrobianos. En orina están asociados a catéteres urinarios permanentes. Reportamos una elevada sensibilidad para tigecilina, importante en el tratamiento de peritonitis secundaria comunitaria o nosocomial por Acinetobacter baumannii "multirresistentes" (MDR) y para colistina que nos permite un tratamiento eficaz para Acinetobacter baumanii "MDR". Se informan elevadas resistencias a gentamicina, esto no concuerda con nuestros resultados ${ }^{27,28}$. Gentamicina por vía inhalatoria alcanza buenas concentraciones en el árbol bronquial con menores efectos secundarios que por vía sistémica ${ }^{27,28}$. Meropenen aún es una buena opción terapéutica. 


\begin{tabular}{|c|c|c|c|c|c|c|c|c|c|c|c|}
\hline \multirow[t]{3}{*}{ Bacteria } & \multirow{3}{*}{$\begin{array}{l}\text { Total de } \\
\text { Muestras }\end{array}$} & TIG & LIN & COL & VAN & MER & ERT & IMI & GEN & MOX & AMI \\
\hline & & \# & \# & \# & \# & \# & \# & \# & \# & \# & \# \\
\hline & & $\mathrm{S} \%$ & $\mathrm{~S} \%$ & $\mathrm{~s} \%$ & $\mathrm{~S} \%$ & $\mathrm{~S} \%$ & $\mathrm{~S} \%$ & $\mathrm{~S} \%$ & $\mathrm{~S} \%$ & $\mathrm{~S} \%$ & $\mathrm{~S} \%$ \\
\hline Staphylococcus & 269 & 266 & 257 & 266 & 269 & 269 & 269 & 266 & 269 & 269 & 224 \\
\hline aureus & & 100 & 100 & 0 & 99 & 13 & 13 & 13 & 0 & 59 & 0 \\
\hline Escherichia & 224 & 95 & 0 & 95 & 0 & 221 & 221 & 222 & 222 & 223 & 221 \\
\hline Coli & & 99 & $\mathrm{NE}$ & 99 & $\mathrm{NE}$ & 94 & 94 & 95 & 50 & 20 & 64 \\
\hline Acinetobacter & 150 & 71 & 0 & 71 & 0 & 15 & 15 & 141 & 141 & 54 & 0 \\
\hline Baumannii & & 96 & $\mathrm{NE}$ & 96 & NE & 80 & 0 & 18 & 94 & 17 & NE \\
\hline Klebsiella & 134 & 80 & 0 & 80 & 0 & 134 & 134 & 134 & 132 & 134 & 129 \\
\hline Pneumoniae & & 94 & $\mathrm{NE}$ & 94 & $\mathrm{NE}$ & 91 & 91 & 92 & 46 & 31 & 74 \\
\hline Pseudomonas & 109 & 49 & 0 & 49 & 0 & 64 & 64 & 106 & 100 & 91 & 100 \\
\hline Aeruginosa & & 0 & NE & 99 & $\mathrm{NE}$ & 42 & 0 & 25 & 33 & 0 & 38 \\
\hline Staphylococcus & 85 & 84 & 83 & 84 & 85 & 85 & 85 & 85 & 85 & 85 & 61 \\
\hline Epidermidis & & 100 & 100 & 0 & 94 & 5 & 5 & 5 & 0 & 65 & 0 \\
\hline Enterococcus & 36 & 31 & 32 & 31 & 36 & 33 & 33 & 31 & 0 & 0 & 36 \\
\hline Faecium & & 100 & 100 & 0 & 36 & 0 & 0 & 0 & $\mathrm{NE}$ & NE & 0 \\
\hline Staphylococcus & 32 & 32 & 31 & 32 & 32 & 32 & 32 & 32 & 32 & 32 & 22 \\
\hline Homini & & 100 & 100 & 0 & 100 & 6 & 6 & 6 & 0 & 66 & 0 \\
\hline Enterococcus & 31 & 24 & 25 & 24 & 31 & 31 & 31 & 31 & 0 & 0 & 29 \\
\hline Faecalis & & 100 & 100 & 0 & 58 & 48 & 48 & 0 & $\mathrm{NE}$ & NE & 0 \\
\hline Staphylococcus & 29 & 26 & 28 & 26 & 29 & 29 & 29 & 29 & 29 & 29 & 25 \\
\hline Haemolyticus & & 100 & 100 & 0 & 100 & 7 & 7 & 7 & 0 & 72 & 0 \\
\hline
\end{tabular}

TIG: Tigeciclina, LIN: Linezolid, COL: Colistina, VAN: Vancomicina, MER: Meropenem, IMI: Imipenem, GEN. Gentamicina, MOX. Moxifloxacino, AMI: Amikacina. \#: Numero de muestras estudiadas, S\%: Porcentaje de sensibilidad, NE: No Estudiado

Tabla 3. Sensibilidad de las bacterias según antibiótico.

Reportamos una sensibilidad baja para imipenem y ampicilina/ sulbactam, lo cual no concuerda a las recomendaciones que indican a estos como de elección. El principal mecanismo de resistencia fue la producción de carbapenemasa y el nivel de resistencia fue "MDR" superior a lo reportado ${ }^{27,28}$.

\section{Pseudomona Auriginosa}

Causa entre $1.8 \%$ a $8.3 \%$ de neumonías comunitarias graves que ocasionan ingreso a "UCl" cuya letalidad va del $50 \%$ al $100 \%$. Son la tercera causa de infecciones urinarias adquiridas asociadas a catéter. La sensibilidad para cefepime, ceftazidima, aztreonam, imipenem, meropenem, ciprofloxacina y gentamicina fueron muy bajas contrario a otros reportes. La sensibilidad para colistina fue elevada, lo cual nos permitirá aun un efectivo tratamiento contra cepas "MDR" y "XDR". Los mecanismos de resistencia más frecuentes fueron: "BLEE", productores de carbapenemasas y betalactamasas tipo AMPc 10,11,29,30

Controlamos el sesgo de selección utilizando un "método de precisión" con criterios de inclusión basados en definiciones internacionales, para el perfil bacteriano se usaron las defini- ciones de la "SEIMC" y para el shock séptico los parámetros The Third International Consensus Definitions for Sepsis and Septic Shock, lo cual aseguro la representatividad de nuestra muestra. El uso de un muestreo no probabilístico de casos consecutivos nos aseguró una adecuada proporción de exámenes para el análisis final. Para evitar los sesgos de medición, debido a datos retrospectivos, usamos un "método de estandarización". Los datos fueron recolectados por médicos especialistas con experiencia en la evaluación de cultivos y antibiogramas que unificaron su metodología. Sin embargo, no se pudo evitar las limitaciones por el menor número de muestras procesadas el 2014 por disminución en el abastecimiento de reactivos. Tampoco pudimos determinar los genes de resistencia en gérmenes "MDR", "XDR", "VISA" y productores de carbapenemasas, ni tuvimos control sobre la toma de cultivos. Para evitar los sesgos de confusión se utilizaron criterios de exclusión basado en un "método de restricción" que evito el ingreso de muestras repetidas o de mala calidad. Los resultados del presente estudio descriptivo trasversal podrían extrapolarse a otras "UCls" de los andes ubicados por encima de los 3,000 "msnm" en pacientes residentes de la altitud que cursan con shock séptico 


\begin{tabular}{|c|c|c|c|c|c|c|c|c|c|c|c|}
\hline \multirow[t]{3}{*}{ Bacteria } & \multirow{3}{*}{$\begin{array}{l}\text { Total, de } \\
\text { Muestras }\end{array}$} & NIT & TET & TS & TOB & LEV & CIP & CEP & AZT & CEF & AS \\
\hline & & $\#$ & $\#$ & $\#$ & $\#$ & $\#$ & $\#$ & $\#$ & $\#$ & $\#$ & $\#$ \\
\hline & & S\% & $S \%$ & S\% & $S \%$ & $\mathbf{S} \%$ & S\% & S\% & S\% & S\% & S\% \\
\hline Staphylococcus & 269 & 269 & 269 & 269 & 72 & 269 & 269 & 245 & 0 & 81 & 245 \\
\hline aureus & & 0 & 6 & 90 & 0 & 15 & 10 & 12 & NE & 0 & 12 \\
\hline Escherichia & 224 & 204 & 221 & 220 & 208 & 222 & 223 & 224 & 156 & 222 & 221 \\
\hline Coli & & 80 & 0 & 2 & 39 & 20 & 19 & 18 & 0 & 17 & 14 \\
\hline Acinetobacter & 150 & 118 & 11 & 141 & 119 & 148 & 150 & 141 & 144 & 133 & 141 \\
\hline Baumannii & & 0 & 0 & 0 & 0 & 0 & 0 & 0 & 0 & 0 & 3 \\
\hline Klebsiella & 134 & 107 & 7 & 132 & 110 & 128 & 133 & 133 & 134 & 130 & 133 \\
\hline Pneumoniae & & 46 & 0 & 0 & 26 & 32 & 31 & 20 & 18 & 18 & 19 \\
\hline Pseudomonas & 109 & 78 & 48 & 98 & 80 & 106 & 106 & 100 & 14 & 85 & 108 \\
\hline Aeruginosa & & 0 & 0 & 0 & 46 & 23 & 20 & 29 & 0 & 29 & 0 \\
\hline Staphylococcus & 85 & 85 & 85 & 85 & 16 & 85 & 85 & 71 & 0 & 23 & 71 \\
\hline Epidermidis & & 100 & 20 & 47 & 0 & 20 & 15 & 31 & $N E$ & 0 & 3 \\
\hline Enterococcus & 36 & 36 & 36 & 0 & 9 & 36 & 36 & 29 & 0 & 9 & 30 \\
\hline Faecium & & 31 & 0 & NE & 0 & 0 & 0 & 0 & $N E$ & 0 & 3 \\
\hline Staphylococcus & 32 & 32 & 32 & 32 & 4 & 32 & 32 & 27 & 0 & 6 & 27 \\
\hline Homini & & 100 & 44 & 53 & 0 & 25 & 9 & 7 & NE & 0 & 7 \\
\hline Enterococcus & 31 & 31 & 31 & 0 & 11 & 31 & 31 & 28 & 0 & 11 & 28 \\
\hline Faecalis & & 68 & 0 & NE & 0 & 19 & 0 & 0 & $N E$ & 0 & 50 \\
\hline Staphylococcus & 29 & 29 & 29 & 29 & 8 & 29 & 29 & 25 & 0 & 10 & 25 \\
\hline Haemolyticus & & 100 & 24 & 34 & 0 & 14 & 7 & 8 & NE & 0 & 8 \\
\hline
\end{tabular}

NIT. Nitrofurantoina, TET: Tetraciclina, TS: Trimetoprim/Sulfametoxazol, TOB: Tobramicina, LEV: Levofloxacino, CIP: Ciprofloxacino, CEP: Cefepima, AZT. Aztreonam, CEF. Ceftazidima, AS. Ampicilina/Sulbactam. \#: Numero de muestras estudiadas, S\%: Porcentaje de sensibilidad, NE: No Estudiado Tabla 4. Sensibilidad de las bacterias según antibiótico.

ya que se realizó durante un periodo de 07 años en un hospital ubicado a 3,250 "msnm". Entre nuestras fortalezas destacamos que describimos la positividad de los cultivos asociados a un análisis bacteriano del mecanismo de resistencia, dato no observado en otros estudios. La relevancia de nuestro estudio se sustenta en la descripción de la realidad bacteriológica de una "UCl" ubicado por encima de los 3,000 "msnm" con una muestra grande que no distan de reportes mundiales que pone en evidencia una común problemática, el uso inadecuado de antibióticos generadores de multirresistencia.

\section{Conclusiones}

Encontramos: a) Un mayor porcentaje de bacterias gramnegativas. Escherichia coli fue la bacteria gramnegativa más frecuente y Staphylococcus aureus el grampositivo b) El cultivo positivo más frecuente estuvo relacionado a muestras del tracto respiratorio inferior. c) Dentro de las bacterias gramnegativas, Pseudomona aeruginosa mostro elevada sensibilidad solo para colistina, el resto también para tigecilina. El mecanismo de resistencia más frecuente fue betalactamasa de espectro extendido. Las Bacterias grampositivas tienen una elevada sensibilidad para tigecilina, linezolid y vancomicina. Su mecanismo de resistencia estuvo relacionado a una elevada resistencia para la ampicilina/sulbactam. d) No encontramos diferencias de los perfiles bacterianos informados en la altitud y los del nivel del mar. Conocer el diagnostico bacteriológico, la sensibilidad y la resistencia bacteriana a los antibióticos en el shock séptico nos dará un parámetro para la elección del inicio de la terapia antibiótica empírica dentro de la "UCl" y colaborar en la disminución de la mortalidad de estos pacientes críticos. Este estudio inicial será el primer paso para generar estudios prospectivos posteriores. Recomendamos a) Tomar en cuenta estos resultados para elegir empíricamente los antibióticos frente a un paciente que ingrese a "UCl" con shock séptico y b) Confirmar nuestros resultados de una elevada sensibilidad "in vitro" de Tigeciclina con otros estudios analíticos.

\section{Referencias bibliográficas}

1. Baykara N, Akalın H, Arslantaş MK, Hancı V, Çağlayan Ç, Kahveci $F$, et al. Epidemiology of sepsis in intensive care units in Turkey: a multicenter, point-prevalence study. Crit Care. 2018;22(1):93107.

2. Martin GS. Sepsis, severe sepsis and septic shock: changes in incidence, pathogens and outcomes. Expert Rev Anti Infect Ther. 2012;10(6):701-6.

3. Vincent J-L, Rello J, Marshall J, Silva E, Anzueto A, Martin CD, et al. International study of the prevalence and outcomes of infection in intensive care units. JAMA. 2009;302(21):2323-9. 


\begin{tabular}{|c|c|c|c|c|c|c|c|c|c|}
\hline & \multicolumn{2}{|c|}{$\begin{array}{c}\text { Ampicilina } \\
\text { Sulbactam } \\
(\# / \%)\end{array}$} & \multicolumn{2}{|c|}{$\begin{array}{l}\text { Meticilina }\left({ }^{\star}\right) \\
(\# / \%)\end{array}$} & \multicolumn{3}{|c|}{$\begin{array}{l}\text { Vancomicina } \\
(\# / \%)\end{array}$} & \multicolumn{2}{|c|}{$\begin{array}{l}\text { Linezolid } \\
(\# / \%)\end{array}$} \\
\hline & s & $\mathbf{R}$ & s & $\mathbf{R}$ & s & $I$ & $R$ & $\mathbf{s}$ & $\mathbf{R}$ \\
\hline \multicolumn{10}{|l|}{ Enterococcus } \\
\hline $\begin{array}{l}\text { Enterococcus } \\
\text { faecium }\end{array}$ & $\begin{array}{c}1 \\
(3 \%)\end{array}$ & $\begin{array}{c}29 \\
(97 \%)\end{array}$ & $\begin{array}{c}0 \\
(0 \%)\end{array}$ & $\begin{array}{c}9 \\
(100 \%)\end{array}$ & $\begin{array}{c}13 \\
(36 \%)\end{array}$ & $\begin{array}{c}0 \\
(0 \%)\end{array}$ & $\begin{array}{c}23 \\
(64 \%)\end{array}$ & $\begin{array}{c}32 \\
(100 \%)\end{array}$ & $\begin{array}{c}0 \\
(0 \%)\end{array}$ \\
\hline \multirow[t]{2}{*}{$\begin{array}{l}\text { Enterococcus } \\
\text { faecalis }\end{array}$} & $\begin{array}{c}14 \\
(50 \%)\end{array}$ & $\begin{array}{c}14 \\
(50 \%)\end{array}$ & $\begin{array}{c}0 \\
(0 \%)\end{array}$ & $\begin{array}{c}11 \\
(100 \%)\end{array}$ & $\begin{array}{c}18 \\
(58 \%)\end{array}$ & $\begin{array}{c}0 \\
(0 \%)\end{array}$ & $\begin{array}{c}13 \\
(42 \%)\end{array}$ & $\begin{array}{c}25 \\
(100 \%)\end{array}$ & $\begin{array}{c}0 \\
(0 \%)\end{array}$ \\
\hline & & & MSSA & MRSA & & VISA & VRSA & & \\
\hline \multicolumn{10}{|l|}{$\begin{array}{r}\text { Staphylococcus } \\
\text { Coagulasa } \\
\text { Positivo }\end{array}$} \\
\hline $\begin{array}{l}\text { Staphylococcus } \\
\text { aureus }\end{array}$ & $\begin{array}{c}30 \\
(12 \%)\end{array}$ & $\begin{array}{c}215 \\
(88 \%)\end{array}$ & $\begin{array}{c}35 \\
(13 \%)\end{array}$ & $\begin{array}{c}234 \\
(87 \%)\end{array}$ & $\begin{array}{c}267 \\
(99 \%)\end{array}$ & $\begin{array}{c}2 \\
(1 \%)\end{array}$ & $\begin{array}{c}0 \\
(0 \%)\end{array}$ & $\begin{array}{c}257 \\
(100 \%)\end{array}$ & $\begin{array}{c}0 \\
(0 \%)\end{array}$ \\
\hline \multicolumn{10}{|l|}{$\begin{array}{r}\text { Staphylococcus } \\
\text { Coagulasa } \\
\text { Negativo }\end{array}$} \\
\hline $\begin{array}{l}\text { Staphylococcus } \\
\text { epidermidis }\end{array}$ & $\begin{array}{c}2 \\
(3 \%)\end{array}$ & $\begin{array}{c}69 \\
(97 \%)\end{array}$ & $\begin{array}{c}0 \\
(0 \%)\end{array}$ & $\begin{array}{c}23 \\
(100 \%)\end{array}$ & $\begin{array}{c}80 \\
(94 \%)\end{array}$ & $\begin{array}{c}2 \\
(2 \%)\end{array}$ & $\begin{array}{c}3 \\
(4 \%)\end{array}$ & $\begin{array}{c}83 \\
(100 \%)\end{array}$ & $\begin{array}{c}0 \\
(0 \%)\end{array}$ \\
\hline $\begin{array}{l}\text { Staphylococcus } \\
\text { hominis }\end{array}$ & $\begin{array}{c}2 \\
(7 \%)\end{array}$ & $\begin{array}{c}25 \\
(93 \%)\end{array}$ & $\begin{array}{c}0 \\
(0 \%)\end{array}$ & $\begin{array}{c}4 \\
(100 \%)\end{array}$ & $\begin{array}{c}32 \\
(100 \%)\end{array}$ & $\begin{array}{c}0 \\
(0 \%)\end{array}$ & $\begin{array}{c}0 \\
(0 \%)\end{array}$ & $\begin{array}{c}31 \\
(100 \%)\end{array}$ & $\begin{array}{c}0 \\
(0 \%)\end{array}$ \\
\hline $\begin{array}{l}\text { Staphylococcus } \\
\text { haemolyticus }\end{array}$ & $\begin{array}{c}2 \\
(8 \%)\end{array}$ & $\begin{array}{c}23 \\
(92 \%)\end{array}$ & $\begin{array}{c}0 \\
(0 \%)\end{array}$ & $\begin{array}{c}8 \\
(100 \%)\end{array}$ & $\begin{array}{c}29 \\
(100 \%)\end{array}$ & $\begin{array}{c}0 \\
(0 \%)\end{array}$ & $\begin{array}{c}0 \\
(0 \%)\end{array}$ & $\begin{array}{c}28 \\
(100 \%)\end{array}$ & $\begin{array}{c}0 \\
(0 \%)\end{array}$ \\
\hline
\end{tabular}

Tabla 5. Mecanismos de Resistencia de las Grampositivas.

\begin{tabular}{|c|c|c|c|c|c|c|}
\hline & \multicolumn{4}{|c|}{ MECANISMOS DE RESISTENCIA } & \multicolumn{2}{|c|}{$\begin{array}{c}\text { NIVELES DE } \\
\text { RESISTENCIA }\end{array}$} \\
\hline & \multicolumn{2}{|c|}{ BETALACTAMASA } & \multirow[t]{2}{*}{ CARBAPENEMASA } & \multirow{2}{*}{$\begin{array}{l}\text { CIERRE DE } \\
\text { PORINAS }\end{array}$} & \multirow[t]{2}{*}{ XDR } & \multirow[t]{2}{*}{ MDR } \\
\hline & BLEE & AMPc & & & & \\
\hline $\begin{array}{l}\text { Escherichia } \\
\text { Coli }\end{array}$ & $172(77 \%)$ & & $12(5 \%)$ & & & \\
\hline $\begin{array}{l}\text { Klebsiella } \\
\text { pneumoniae }\end{array}$ & $56(42 \%)$ & & & & $9(7 \%)$ & \\
\hline $\begin{array}{l}\text { Acinetobacter } \\
\text { baumanni }\end{array}$ & & & $116(77 \%)$ & & $3(2 \%)$ & $132(88 \%)$ \\
\hline $\begin{array}{l}\text { Pseudomonas } \\
\text { aeriginosa }\end{array}$ & $99(91 \%)$ & $24(22 \%)$ & $79(72 \%)$ & $4(4 \%)$ & & \\
\hline
\end{tabular}

Tabla 6. Mecanismos de Resistencia de los Bacilos Gramnegativas.

4. Rhodes A, Evans LE, Alhazzani W, Levy MM, Antonelli M, Ferrer $\mathrm{R}$, et al. Surviving Sepsis Campaign: International Guidelines for Management of Sepsis and Septic Shock: 2016. Intensive Care Med. 2017;43(3):304-77.

5. Cisneros-Herreros M, Cobo-Reinoso J, Pujol-Rojo M, Rodríguez-Baño J, Salavert-Lletí M. Guía para el diagnóstico y tratamiento del paciente con bacteriemia. Guías de la Sociedad Española de Enfermedades Infecciosas y Microbiología Clínica (SEIMC). Enfermedades Infecciosas y Microbiología Clínica. 2007;25(2):111-30.

6. Paul M, Shani V, Muchtar E, Kariv G, Robenshtok E, Leibovici L. Systematic review and meta-analysis of the efficacy of appropriate empiric antibiotic therapy for sepsis. Antimicrob Agents Chemother. 2010;54(11):4851-63.

7. Singer M, Deutschman CS, Seymour CW, Shankar-Hari M, Annane D, Bauer M, et al. The Third International Consensus Definitions for Sepsis and Septic Shock (Sepsis-3). JAMA. 2016;315(8):801-10.

8. Bennett J, Dolin R, Blaser MJ. Mandell, Douglas y Bennett. Enfermedades infecciosas. Síndrome de inmunodeficiencia adquirida. 8th Edition. Elsevier; 288 p.
9. Tinoco-Solórzano A, Nieto Estrada VH, Vélez-Páez JL, Molano Franco D, Viruez Soto A, Villacorta-Córdova F, et al. Medicina intensiva en la altitud. Revisión de alcance. Intensivos. 2020;13(4):218-25.

10. Molina FJ, Díaz CA, Barrera L, Rosa GDL, Dennis R, Dueñas C, et al. Perfil microbiológico de la Infecciones en Unidades de Cuidados Intensivos de Colombia (EPISEPSIS Colombia). Medicina Intensiva. 2011;35(2):75-83.

11. Gómez-González JF, Sánchez-Duque JA. Perfil microbiológico y resistencia bacteriana en una unidad de cuidados intensivos de Pereira, Colombia, 2015. Medicas UIS. 2018;31(2):9-15.

12. Paz Rojas E, Ponce de León Pandolfi D, Ramírez Ponce R. Resistencia bacteriana en cuidados intensivos y tendencia actual: Departamento de Cuidados Críticos, Servicio de Cuidados Intensivos del Hospital Nacional Guillermo Almenara Irigoyen, Essalud, Lima, Perú, 2004-2006. Acta Médica Peruana. 2008;25(3):140-7.

13. Fernández-Mogollón J, Tello-Vera S, Pizarro-Chima F. Perfil Microbiológico de un Hospital del Seguro Social Nivel III, Chiclayo-Perú. 2014. Revista del Cuerpo Médico del HNAAA. 2016;9(1):6-13. 
14. Quintanilla Chanez JA, Orellana Vicentes R, Alfaro Claros C. Perfil Microbiológico de Infecciones Nosocomiales en la Unidad de Terapia Intensiva del Hospital Clínico Viedma. Gaceta Médica Boliviana. 2011;34(1):37-9.

15. Alvarez C, Cortes J, Arango Á, Correa C, Leal A. Resistencia Antimicrobiana en Unidades de Cuidado Intensivo de Bogotá, Colombia, 2001-2003. Revista de Salud Pública. 2006;Sup 8(1):86-101.

16. Vélez J, Buchelo R, Montalvo M, Gallardo F, Velez P, Escobar C Epidemiological monitoring with routine rectal swabs. Rev ecuat med Eugenio Espejo. 2016;5(7):43-6.

17. Bauer PR, Sampathkumar P. Methicillin-Resistant Staphylococcus aureus Infection in ICU: What Is the Best Prevention Strategy? Crit Care Med. 2017;45(8):1413-4.

18. Shariati A, Dadashi M, Chegini Z, Van Belkum A, Mirzaii M, Khoramrooz SS, et al. The global prevalence of Daptomycin, Tigecycline, Quinupristin/Dalfopristin, and Linezolid-resistant Staphylococcus aureus and coagulase-negative staphylococci strains: a systematic review and meta-analysis. Antimicrob Resist Infect Control. 2020;9(1):56-76.

19. López-Fabal F, Román Alonso F, Almagro Moltó M, Sanz Rodríguez N, Gómez-Garcés JL. Staphylococcus coagulasa negativos resistentes al linezolid: características fenotípicas, genotípicas y sensibilidad a combinaciones de antibióticos. Enfermedades Infecciosas y Microbiología Clínica. 2013;31(7):442-7.

20.Cercenado E. Epidemiología de la infección por grampositivos resistentes. Rev esp quimioter. 2016;29(supl.1):6-9.

21. Pfaller MA, Cormican M, Flamm RK, Mendes RE, Jones RN. Temporal and Geographic Variation in Antimicrobial Susceptibility and Resistance Patterns of Enterococci: Results From the SENTRY Antimicrobial Surveillance Program, 1997-2016. Open Forum Infectious Diseases. 2019;6(Supplement_1):S54-62.

22. Molano-Franco D, Villabón $M$, Gómez $M$, Muñoz L, Beltrán $E$, Barbosa $F$, et al. Determinación de los factores de riesgo en sepsis por Enterococcus vancomicino resistente. Estudio de casos y controles en pacientes críticamente enfermos. Infectio 2020;24(4):248-54.
23. Salame-Khouri L, Contreras-Pichardo B, Arias-Rodríguez S, Mondragón-Soto M, Cataneo-Serrato JL, Núñez-Martínez M, et al. Epidemiología de las bacteriemias por Escherichia coli en dos hospitales de tercer nivel de la Ciudad de México. An Med Asoc Med Hosp ABC. 2018;63(2):91-5.

24. Restrepo-Álvarez CA, Bernal E, Ascuntar-Tello J, Jaimes F, Restrepo-Álvarez CA, Bernal E, et al. Análisis clínico y microbiológico de la sepsis grave y el choque séptico por Escherichia coli en Medellín, Colombia. Revista chilena de infectología. 2019:36(4):447-54.

25. Taraghian A, Nasr Esfahani B, Moghim S, Fazeli H. Characterization of Hypervirulent Extended-Spectrum $囚$-Lactamase-Producing Klebsiella pneumoniae Among Urinary Tract Infections: The First Report from Iran. Infect Drug Resist. 2020;13:3103-11.

26. Soria-Segarra C, Soria-Segarra C, Catagua-González A, Gutiérrez-Fernández J. Carbapenemase producing Enterobacteriaceae in intensive care units in Ecuador: Results from a multicenter study. J Infect Public Health. 2020;13(1):80-8.

27. Piperaki E-T, Tzouvelekis LS, Miriagou V, Daikos GL. Carbapenem-resistant Acinetobacter baumannii: in pursuit of an effective treatment. Clin Microbiol Infect. 2019;25(8):951-7.

28. Máiz Carro L, Blanco-Aparicio M. Nuevos antibióticos inhalados y formas de administración. Open Respiratory Archives. 2020;2(3):251-64.

29.Paz-Zarza VM, Mangwani-Mordani S, Martínez-Maldonado A, Álvarez-Hernández D, Solano-Gálvez SG, Vázquez-López R. Pseudomonas aeruginosa: Pathogenicity and antimicrobial resistance in urinary tract infection. Rev Chilena Infectol. 2019;36(2):180-9.

30. Angles-Yanqui E, Chumbes-Pérez J, Huaringa-Marcelo J. Colistin-based treatment for extensively drug resistant (XDR) Pseudomonas aeruginosa and Acinetobacter baumannii infection at a third level hospital. Infectio. 2020;24(4):201-7.

Received: 6 Mayo 2021

Accepted: 27 Octubre 202 